\title{
Modeling spin interactions in carbon peapods using a hybrid density functional theory
}

\author{
Ling Ge, ${ }^{1, *}$ Barbara Montanari, ${ }^{2}$ John H. Jefferson, ${ }^{3}$ David G. Pettifor, ${ }^{1}$ Nicholas M. Harrison,,${ }^{2,4}$ and G. Andrew D. Briggs ${ }^{1}$ \\ ${ }^{1}$ Department of Materials, University of Oxford, Oxford OX1 3PH, United Kingdom \\ ${ }^{2}$ STFC Rutherford Appleton Laboratory, Didcot, Oxfordshire OX11 OQX, United Kingdom \\ ${ }^{3}$ Sensors and Electronics Division, QinetiQ, St. Andrews Road, Malvern, Worcestershire WR14 3PS, United Kingdom \\ ${ }^{4}$ Department of Chemistry, Imperial College London, London SW7 2AZ, United Kingdom \\ (Received 6 March 2008; revised manuscript received 19 May 2008; published 12 June 2008)
}

\begin{abstract}
The Sc@ $\mathrm{C}_{82}$ endohedral fullerenes inside a single-wall semiconducting or metallic nanotube form a welldefined chain of antiferromagnetically coupled spins. Using hybrid density functional theory (DFT), we find that the spin resides mainly on the fullerene cage, whether or not the fullerenes are in a nanotube. The spin interactions decay exponentially with fullerene separation and the system can be described by a simple antiferromagnetic Heisenberg spin chain. Energy parameters for a generalized Hubbard-Anderson model are deduced from the DFT calculations and yield a second-order Heisenberg exchange energy, which is in good agreement with total-energy calculations for parallel and antiparallel spin configurations. Within the accuracy of the calculations, neither semiconducting nor metallic nanotubes affect the interactions between the fullerene electron spins.
\end{abstract}

DOI: 10.1103/PhysRevB.77.235416 PACS number(s): 73.61.Wp, 03.67.-a, 71.15.Mb, 73.63.Fg

\section{INTRODUCTION}

Spin chains have potential for controlled interactions ${ }^{1}$ for quantum computing. Carbon is a candidate host for spin qubits because in ${ }^{12} \mathrm{C}$ materials, the small spin-orbit coupling and the absence of hyperfine coupling ensure long spin coherence times. Carbon peapods, ${ }^{2}$ which are single-walled carbon nanotubes (SWNTs) containing fullerenes, have been proposed as hosts for spin qubits. ${ }^{3}$ The fabrication of nanoscale electronic devices, such as field effect transistors with carbon peapods containing various endohedral fullerenes, is well established. ${ }^{4}$ When spin active metallic atoms such as Sc are incarcerated in a carbon cage, the system develops hybridized orbitals resulting in an unpaired electron delocalized across the fullerene cage. ${ }^{5}$ Here we report on detailed numerical simulations that establish the nature of the spinspin interactions both between endohedral fullerenes and between fullerenes and nanotubes. The dominant interaction is of the Heisenberg form, which has potential for quantum computing in one-dimensional chains. ${ }^{6}$ Quantum computing protocols have been devised and experimentally demonstrated, which allow chains of identical units to be controlled globally, circumventing the difficulty in local addressing. ${ }^{7}$ It is necessary to be able to set and measure the qubits at the end of the chain, but all the other qubits in the chain can be manipulated collectively without needing to target them individually. In this way, information can be propagated and processed in the spin chain and scalable quantum computation can be performed. Even without long-range antiferromagnetic order, quantum computation is still possible with global addressing given that there are strong antiferromagnetic correlations. ${ }^{7}$ A well-defined initial state can be achieved by algorithmical cooling. ${ }^{8}$ However, there are several hurdles to overcome before such systems can be realistically considered as a basic technology for quantum computation. Open experimental challenges include the control and measurement of spins without affecting the neighboring spins in the global control protocol, the engineering of the intermolecular coupling strength, and the measurement of the decoherence time of a single spin on the fullerene cage.

Four fundamental problems need to be understood in order to demonstrate well-defined qubits in carbon peapods as proposed in Ref. 9: (i) the charge arrangement within the carbon peapods, (ii) the electron-spin distribution, (iii) the coupling between spin qubits, and (iv) the nature of the spin interactions between fullerenes and nanotube. Density functional theory (DFT) calculations have been reported on several model systems, such as SWNTs containing $\mathrm{C}_{60}, \mathrm{~K}_{x} \mathrm{C}_{60}$, $\mathrm{Y} @ \mathrm{C}_{60}, \mathrm{C}_{82}$, La@ $\mathrm{C}_{82}, \mathrm{La}_{2} @ \mathrm{C}_{82}$, and $\mathrm{Sc}_{3} \mathrm{~N} @ \mathrm{C}_{80} .{ }^{10-17} \mathrm{To}$ date little work has been done on understanding the spin properties of peapods and the above mentioned issues (ii), (iii), and (iv) have not been addressed in detail thus far. We have computed the charge and spin distributions and the electronic structures within $\mathrm{Sc} @ \mathrm{C}_{82}$ chains and peapod structures. We find well-defined spin-1/2 qubits on the fullerenes with strong evidence for a nearest-neighbor Heisenberg exchange interaction. In order to describe the influence on the spin qubits localized on the fullerenes from propagating electrons or holes in the nanotube, we need to go beyond DFT to a model which is capable of describing the low-energy charge-spin excitations of the system. We conjecture a generic Hubbard-Anderson model, which has these properties, to estimate the low-energy spin interactions; in particular, the Heisenberg exchange between spins along the fullerene chain and the Kondo exchange interaction between localized spins on the fullerenes and spins of propagating electrons or holes in the nanotube.

\section{SPIN-POLARIZED DENSITY FUNCTIONAL THEORY RESULTS}

\section{A. System's geometry and computational details}

As model systems, we choose Sc@ $\mathrm{C}_{82}$ in $(14,7)$ (semiconducting) and $(11,11)$ (metallic) SWNTs. Our calculations predict an exothermic encapsulation of $\mathrm{Sc} @ \mathrm{C}_{82}$ for both 
(a)

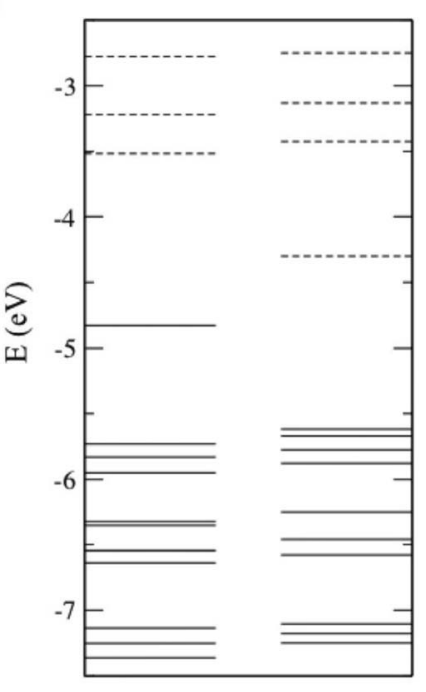

(b)

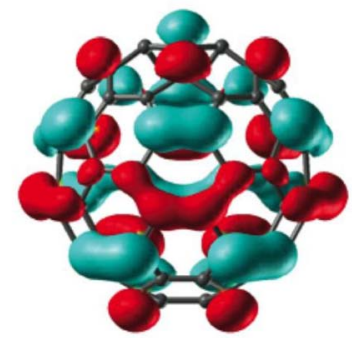

(c)

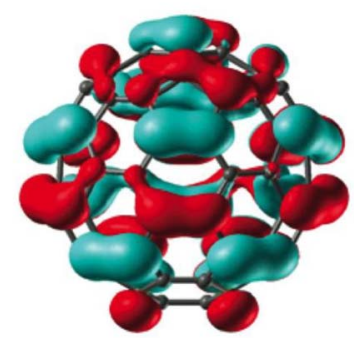

FIG. 1. (Color online) (a) Eigenspectrum of relaxed Sc @ $\mathrm{C}_{82}$ molecule. Left- (right-) hand side refers to spin-up (-down) electrons. The solid (dashed) lines refer to occupied (unoccupied) energy levels. (b) HOMO of relaxed $\mathrm{Sc} @ \mathrm{C}_{82}$. (c) $(\mathrm{LUMO}+1)$ of relaxed $\mathrm{C}_{82}$. The dark gray (red) and light gray (blue) lobes represent positive and negative phases, respectively. tubes. The repeat units containing one $\mathrm{Sc} @ \mathrm{C}_{82}$ molecule in the $(14,7)$ and $(11,11)$ peapods are 11.42 and $12.47 \AA$, respectively. ${ }^{18}$ In order to study the exchange interaction, we use double unit cells containing two $\mathrm{Sc} @ \mathrm{C}_{82}$ molecules. The interwall separations between the $(14,7)$ and $(11,11)$ tubes and $\mathrm{Sc} @ \mathrm{C}_{82}$ are $3.35 \AA$ (van der Waals distance) and $3.55 \AA$, respectively. The relaxed structure of $\mathrm{Sc} @ \mathrm{C}_{82}$ is found to be in agreement with Ref. 5 with a Sc-C distance of $2.26 \AA$. The DFT calculations are performed with the hybrid exchange density functional B3LYP (Refs. 19-21) as implemented in the CRYSTAL package. ${ }^{22}$ The calculations reported here are all electron, i.e., with no shape approximation to the ionic potential or electron charge density. The geometry optimizations are performed using the algorithm proposed by Schlegel et $a l .{ }^{23}$ The crystalline wave functions are expanded in Gaussian basis sets of double valence quality $\left(6-21 G^{*}\right.$ for $\mathrm{C}$ and $864-11 G^{*}$ for $\left.\mathrm{Sc}\right)$. Atomic charges are estimated using Mulliken population analysis. ${ }^{24}$ The system is modeled as a one-dimensionally periodic array with reciprocal space sampling performed on an adaptation of the Monkhorst-Pack grid containing 30 symmetry irreducible $k$ points, which converges the total energy to within $10^{-4} \mathrm{eV}$ per unit cell.

\section{B. Eigenspectrum and wavefunction of Sc@ $\mathrm{C}_{82}$ molecule}

Figure 1(a) shows the calculated electronic eigenspectrum for the relaxed Sc $@ \mathrm{C}_{82}$ molecule. Sc has three valence electrons and the ground state of $\mathrm{Sc} @ \mathrm{C}_{82}$ is found to be a spin$1 / 2$ system. The unpaired electron occupies the highest occupied molecular orbital (HOMO) of $\mathrm{Sc} @ \mathrm{C}_{82}$, which constitutes the spin qubit. The HOMO-lowest unoccupied molecular orbital (LUMO) gap is $0.530 \mathrm{eV}$. The separation between the HOMO and (HOMO-1) is $0.789 \mathrm{eV}$. Therefore, the HOMO is well separated from the energy levels above and below leading to a well-defined qubit. The Sc $@ \mathrm{C}_{82}$ HOMO is delocalized across the fullerene cage as depicted in Fig. 1(b), which is in agreement with the results found in Refs. 5 and 25. Furthermore, we establish that the HOMO of $\mathrm{Sc} @ \mathrm{C}_{82}$ is virtually identical to the (LUMO+1) of $\mathrm{C}_{82}$, as shown in Fig. 1(c), whereas the lower lying orbitals are hybrids of $\mathrm{Sc}$ and $\mathrm{C}_{82}$. Thus $\mathrm{Sc}$ acts as a perfect donor to the $\mathrm{C}_{82}$ cage for the HOMO state.

\section{Electronic charge and spin populations}

Figure 2 shows the electronic charge rearrangement following the $\mathrm{Sc} @ \mathrm{C}_{82}$ encapsulation in the $(14,7)$ nanotube. The charge depletion from the nanotube is concentrated around the fullerene sites. Similar qualitative results are obtained for the $(11,11)$ nanotube, resembling that in La@ $\mathrm{C}_{82} @(17,0) .{ }^{10}$ Table I shows the charge and spin populations in $\quad \mathrm{Sc} @ \mathrm{C}_{82}, \quad \mathrm{Sc} @ \mathrm{C}_{82} @(14,7), \quad$ and Sc@ $\mathrm{C}_{82} @(11,11)$ peapods. In $\mathrm{Sc} @ \mathrm{C}_{82}, 1.64$ electrons transfer from the $\mathrm{Sc}$ atom to the $\mathrm{C}_{82}$ cage indicating a partially covalent Sc-cage bond. In both Sc @ $\mathrm{C}_{82} @(14,7)$ and $\mathrm{Sc} @ \mathrm{C}_{82} @(11,11)$ peapods, electron transfer occurs from the nanotube and the $\mathrm{Sc}$ atom to the $\mathrm{C}_{82}$ cage due to hybridization between the occupied states of the nanotube and fullerenes. The charge transfer from the $\mathrm{Sc}$ atom to the $\mathrm{C}_{82}$ cage in $\mathrm{Sc} @ \mathrm{C}_{82} @(14,7)$ and $\mathrm{Sc} @ \mathrm{C}_{82} @(11,11)$ is very
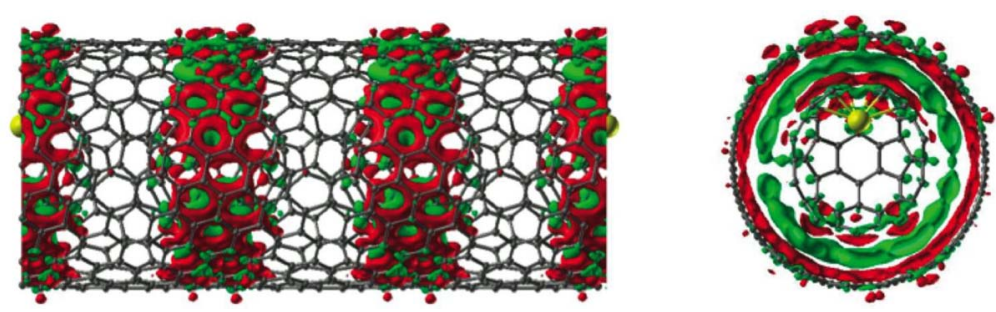

FIG. 2. (Color online) Charge transfer in Sc@ $C_{82} @(14,7)$ peapod. The values for dark gray (red) and light gray (green) surfaces are $\pm 0.001 e / \AA^{3}$. Left (right) hand shows front (side) views. The atom colored in light gray (gold online) is Sc. 
TABLE I. Charge and spin populations in $\mathrm{Sc} @ \mathrm{C}_{82}$, $\mathrm{Sc} @ \mathrm{C}_{82} @(14,7)$, and Sc@ $\mathrm{C}_{82} @(11,11)$ peapods.

\begin{tabular}{lccc}
\hline \hline System & Component & $q(e)$ & $m\left(\mu_{B}\right)$ \\
\hline $\mathrm{Sc} @ \mathrm{C}_{82}$ & $\mathrm{Sc}$ & 1.64 & 0.03 \\
& $\mathrm{C}_{82}$ & -1.64 & 0.97 \\
$\mathrm{Sc} @ \mathrm{C}_{82} @(14,7)$ & $\mathrm{Sc}$ & 1.63 & 0.03 \\
& $\mathrm{C}_{82}$ & -1.76 & 0.97 \\
& Tube & 0.13 & 0.00 \\
$\mathrm{Sc} @ \mathrm{C}_{82} @(11,11)$ & $\mathrm{Sc}$ & 1.63 & 0.03 \\
& $\mathrm{C}_{82}$ & -1.70 & 0.97 \\
& Tube & 0.07 & 0.00 \\
\hline \hline
\end{tabular}

similar to that of $\mathrm{Sc} @ \mathrm{C}_{82}$. The electron-spin distribution is also similar as $97 \%$ of the density resides on the $\mathrm{C}_{82}$ cage and only $3 \%$ on the Sc atom. The shape of the spin-density distribution in the peapods closely resembles that of the HOMO of Sc @ $\mathrm{C}_{82}$ illustrated in Fig. 1(b). The charge transfer and spin distribution are insensitive to encapsulation.

\section{Range of exchange interaction}

In the predicted ground state configuration of the peapods, the spin direction alternates along the $\mathrm{Sc} @ \mathrm{C}_{82}$ chain; the corresponding configuration with parallel spins is higher in energy. We denote these configurations as antiferromagnetic (AF) and ferromagnetic (FM) states, respectively. Both states are found to be Mott insulators and have a total energy lower than the restricted Kohn-Sham solution. For the Sc@ $\mathrm{C}_{82} @(14,7)$ peapod where the interfullerene spacing is $3.42 \AA$, the energy difference between the nonmagnetic and FM states is $0.108 \mathrm{eV} /$ cell. The exchange parameter $J$, defined as the energy difference between FM and AF configurations, is $3 \mathrm{meV}$ per cell (containing two spins). The behavior of $J$ as a function of the interfullerene separation $R$ in a $\mathrm{Sc} @ \mathrm{C}_{82}$ chain is plotted in Fig. 3. The values of $J$ calculated for the peapods at discrete values of $R$, as indicated in Fig. 3, coincide with those obtained for the $\mathrm{Sc} @ \mathrm{C}_{82}$ chain within the accuracy of the present calculations. At these separations, the intermolecular spin interaction is therefore via direct exchange between fullerenes, with a negligible contribution from interactions via the nanotube. This intermolecular coupling is much larger than the classical dipole coupling of $\mathrm{Na} @ \mathrm{C}_{60}{ }^{26}$ and even larger than that computed for defective fullerenes with intercage links, ${ }^{27}$ ensuring $>10^{3}$ two-qubit gate operations within the decoherence time. This surprising result follows from the HOMOs in the $\mathrm{Sc} @ \mathrm{C}_{82}$ chain being very extended as illustrated in Fig. 3 . The $p_{z}$ orbitals on the closest $\mathrm{C}$ atoms belonging to adjacent molecules overlap in a $\sigma$-type fashion. This implies that the exchange interaction could be tuned by varying the separation between the fullerenes in peapods. Such high $J$ values are consistent with recent magnetic susceptibility experiments on $\mathrm{Sc} @ \mathrm{C}_{82}$ solids, ${ }^{28}$ which show AF Curie-Weiss temperature $\simeq 300 \mathrm{~K}$, consistent with $J=17 \mathrm{meV}$ at $R=3.08 \AA$ in Fig. 3, which corresponds to the measured lattice spacing.

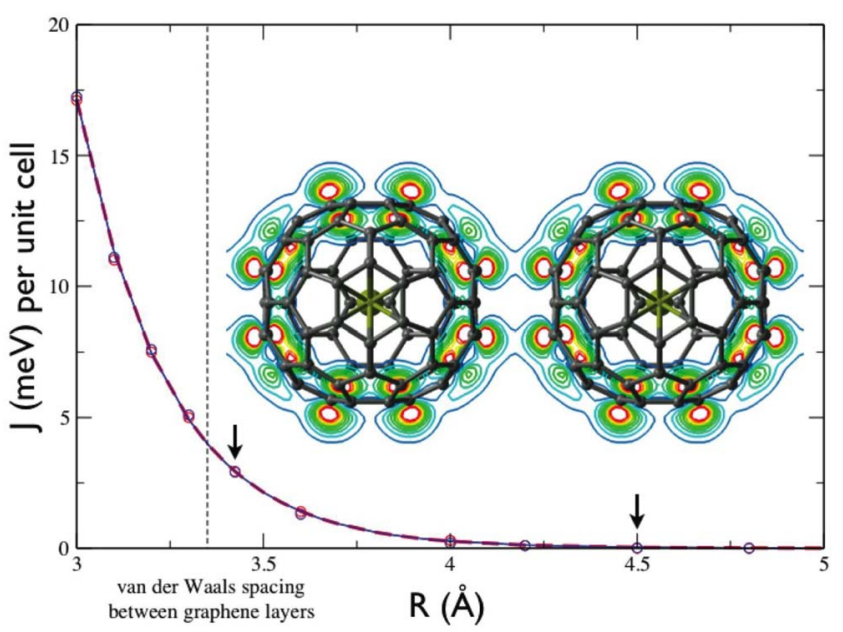

FIG. 3. (Color online) Exchange interaction strength $J$ as a function of interfullerene separation for a chain of $\mathrm{Sc} @ \mathrm{C}_{82}$ fullerenes. The empty circles refer to calculated results and are fitted using exponential decay law (lines) of the form $J_{0} e^{-\lambda\left(R-R_{0}\right)}$, where $J_{0}$ $=4.0 \mathrm{meV}, \lambda=4.16 \AA^{-1}$, and $R_{0}=3.35 \AA$. The dashed (red) line refers to $J$ obtained from the DFT energy difference between FM and AF states. The solid (blue) line refers to $J_{\text {eff }}$ obtained from the Heisenberg model $4 t^{2} / U_{\text {eff }}$, where $U_{\text {eff }}$ is fitted to be $0.412 \mathrm{eV}$ from the value of $J$ at $R_{0}$. The arrows show discrete values of $R$ at which $J$ is calculated for peapods. The inset shows electron density contributed by highest occupied states of the $\mathrm{Sc} @ \mathrm{C}_{82}$ chain. The range of isovalues is $0-0.002 \mathrm{e} / \AA^{3}$.

\section{E. Electronic band structures}

The AF band structures of the peapods are plotted in Fig. 4. They are consistent with a chain of fullerenes interacting weakly with a nanotube. In the semiconducting case [Fig. 4(a)], there are very narrow bands consistent with weak hopping of electrons along the fullerene chain. In the metallic case [Fig. 4(b)], these bands cross the wide nanotube bands. The weak interaction actually opens up small hybridization gaps or anticrossings, as seen in the circle areas in Fig. 4. This will be discussed in detail in Sec. III.

\section{HUBBARD-ANDERSON MODEL}

The AF band structures in Fig. 4 display very narrow but almost perfect cosine form for the bands derived from the HOMO and LUMO of Sc @ $\mathrm{C}_{82}$. This is well described by a simple tight-binding model, as shown in Fig. 5(a), where we have expanded the energy axis for the HOMO/LUMOderived bands and show the fit for perfect cosine dispersions. Similarly, for the metallic nanotube case shown in Fig. 4(b), we again see the narrow cosine bands of the fullerene chain which now anticross the linear-dispersion conduction and valence bands of the nanotube, giving hybridization gaps at the anticrossing points. These results imply that the hybrid system may be described by a Hubbard-Anderson model with parameters chosen so as to approximately reproduce the dispersion curves of Fig. 4 in mean-field theory. However, such a generic Hubbard-Anderson model goes beyond DFT and is capable of describing the low-energy spin and charge excita- 


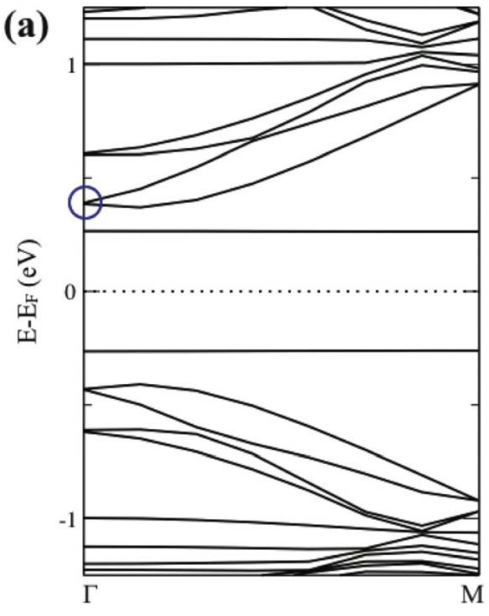

(b)

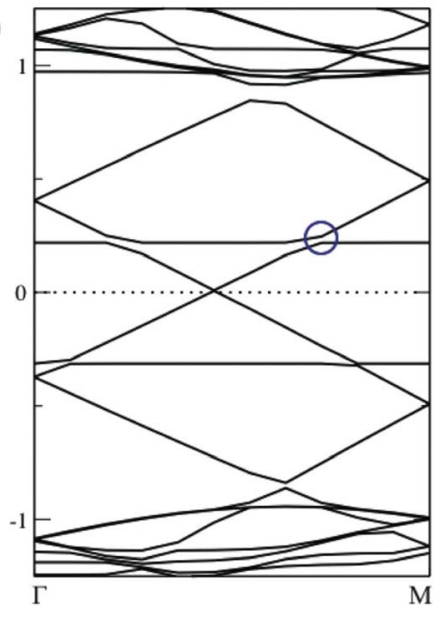

FIG. 4. (Color online) Spin-polarized band structures of the (a) $\mathrm{Sc} @ \mathrm{C}_{82} @(14,7)$ and (b) $\mathrm{Sc} @ \mathrm{C}_{82} @(11,11)$ peapods for the AF configuration. $E_{F}$ is the Fermi energy. tions of the system. The Hubbard-Anderson model may be used directly to estimate the Heisenberg exchange between spins along the fullerene chain and the Kondo exchange interaction between localized spins on the fullerenes and spins of propagating electrons or holes in the nanotube. The Hamiltonian is

$$
H_{H A}=H_{n t}+H_{f c}+H_{\mathrm{int}}
$$

where

$$
\begin{gathered}
H_{n t}=\sum_{l k \sigma} \epsilon_{l k} c_{l k \sigma}^{\dagger} c_{l k \sigma}, \\
H_{f c}=\sum_{k \sigma} E_{k} a_{k \sigma}^{\dagger} a_{k \sigma}+U \sum_{m} n_{m \uparrow} n_{m \downarrow}, \\
H_{\mathrm{int}}=\sum_{l k \sigma} \gamma_{l k}\left[a_{k \sigma}^{\dagger} c_{l k \sigma}+\text { h.c. }\right] .
\end{gathered}
$$

Here, $c_{l k \sigma}^{\dagger}$ is a creation operator for an electron in the nanotube with band index $l$ with quasimomentum $k, a_{k \sigma}^{\dagger}$ is a creation operator for an electron in the HOMO-derived band of the fullerenes, $\gamma_{l k}$ is the hybridization parameter between fullerenes and nanotube bands, $U$ is the intrafullerene Coulomb repulsion energy, and $n_{m}$ is the number operator for the site $m$ of the $\mathrm{Sc} @ \mathrm{C}_{82}$ chain.

The fullerene band derived from the HOMO of Sc @ $\mathrm{C}_{82}$ splits into occupied and unoccupied bands when the Hubbard- $U$ term is treated in mean-field theory. To solve the Schrödinger equation for Hamiltonian (1), with unit cell containing two Sc@ $\mathrm{C}_{82}$ fullerenes labeled 1 and 2, we look for antiferromagnetic solutions in which $\left\langle n_{m 1 \uparrow}\right\rangle \neq\left\langle n_{m 1 \downarrow}\right\rangle$ and neglect intrasite charge fluctuations, i.e., we make the approximation

$$
\left(n_{m 1 \uparrow}-\left\langle n_{m 1 \uparrow}\right\rangle\right)\left(n_{m 1 \downarrow}-\left\langle n_{m 1 \downarrow}\right\rangle\right) \simeq 0,
$$

giving

$$
n_{m 1 \uparrow} n_{m 1 \downarrow} \simeq n_{m 1 \uparrow}\left\langle n_{m 1 \downarrow}\right\rangle+n_{m 1 \downarrow}\left\langle n_{m 1 \uparrow}\right\rangle-\left\langle n_{m 1 \uparrow}\right\rangle\left\langle n_{m 1 \downarrow}\right\rangle,
$$

and similarly for $n_{m 2 \uparrow} n_{m 2 \downarrow}$. For a perfect AF spin chain with

$$
\left\langle n_{m 1 \uparrow}\right\rangle=1,\left\langle n_{m 1 \downarrow}\right\rangle=0,
$$

$$
\left\langle n_{m 2 \uparrow}\right\rangle=0,\left\langle n_{m 2 \downarrow}\right\rangle=1 .
$$

Equation (3) reduces to the mean-field Hamiltonian,

$$
H_{f c}=h_{\uparrow}+h_{\downarrow},
$$

where

$$
\begin{aligned}
h_{\uparrow}= & \sum_{m}\left[\varepsilon_{0} n_{m 1 \uparrow}+\left(\varepsilon_{0}+U\right) n_{m 2 \uparrow}\right. \\
& \left.+t\left(a_{m 1 \uparrow}^{\dagger} a_{m 2 \uparrow}+a_{m 2 \uparrow}^{\dagger} a_{m+1,1 \uparrow}+\text { h.c. }\right)\right],
\end{aligned}
$$

and

$$
a_{m \nu \uparrow}^{\dagger}=\frac{1}{\sqrt{N}} \sum_{k} e^{-i k m} a_{k \nu \uparrow}^{\dagger}(\nu=1,2),
$$

and similarly for $h_{\downarrow}$. Here, $\varepsilon_{0}$ and $t$ are the on-site energy and nearest-neighbor hopping parameter satisfying $E_{k}=\varepsilon_{0}$

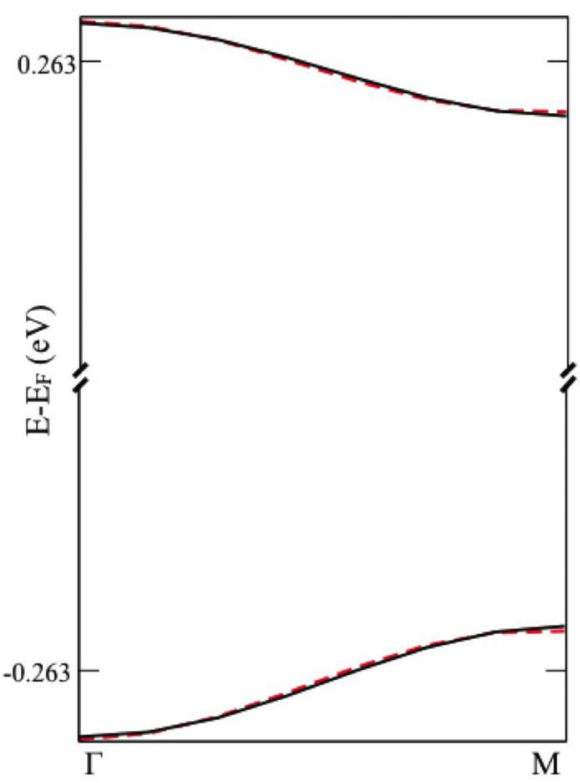

FIG. 5. (Color online) The bands derived from the HOMO/ LUMO of Sc@ $\mathrm{C}_{82}$ in the $\mathrm{Sc} @ \mathrm{C}_{82} @(14,7)$ peapod for the AF configuration in an expanded energy axis. The solid lines refer to DFT results and are fitted using cosine functions (dashed lines). 
$-2 t \cos k$. Transforming back into $k$ space, we get

$$
h_{\uparrow}=\sum_{k}\left(\begin{array}{cc}
\varepsilon_{0} & t\left(1+e^{-i k}\right) \\
t\left(1+e^{i k}\right) & \varepsilon_{0}+U
\end{array}\right)\left(\begin{array}{l}
n_{1 k \uparrow} \\
n_{2 k \uparrow}
\end{array}\right),
$$

giving eigenenergies for both spin-up and spin-down electrons,

$$
\widetilde{E}_{k}^{ \pm}=\varepsilon_{0}+\frac{U}{2} \pm \frac{U}{2} \sqrt{1+\frac{8 t^{2}}{U^{2}}(1+\cos k)}
$$

For $t \ll U$, we obtain the dispersion energies of the bands derived from the HOMO and LUMO of $\mathrm{Sc} @ \mathrm{C}_{82}$,

$$
\begin{gathered}
\widetilde{E}_{k}^{-}=\varepsilon_{0}-2 \widetilde{t}(1+\cos k), \\
\widetilde{E}_{k}^{+}=\varepsilon_{0}+U+2 \widetilde{t}(1+\cos k),
\end{gathered}
$$

where

$$
\tilde{t}=\frac{t^{2}}{U} .
$$

A Hubbard $U \simeq 0.53 \mathrm{eV}$ in Eq. (3) is obtained from the energy gap between centers of the bands derived from the HOMO and LUMO of Sc @ $\mathrm{C}_{82}$ in Fig. 4. This solution is also equivalent to a Stoner model for exchange $I=U$, where $J=4 t^{2} / U{ }^{29}$ The behavior of the computed hopping parameter $t$ as a function of $R$ in a Sc $@ \mathrm{C}_{82}$ chain is fitted by an exponential decay law of the form $t_{0} e^{-\lambda\left(R-R_{0}\right)}$, where $t_{0}$ $=20.3 \mathrm{meV}, \lambda=2.08 \AA^{-1}$, and $R_{0}=3.35 \AA$, showing $t^{2}$ and $J$ scaling in the same way. For the values of $R$ in the $(14,7)$ and $(11,11)$ peapods, $t=17$ and $2 \mathrm{meV}$, respectively.

An effective $U$ is fitted by fixing $J=4 t^{2} / U_{\text {eff }}$ within the Heisenberg model at $R_{0}$, giving $U_{\text {eff }}=0.412 \mathrm{eV}$, in good agreement with the DFT calculations. Figure 3 shows that $J_{\text {eff }}$ and $J$ are indistinguishable, implying approximately constant $U$ over the range of $R$ considered. $U$ deduced from the DFT band gap is consistent with the mean-field solution of the Hubbard-Anderson model and the total energy difference between FM and AF solutions. With $t \ll U$ for the peapods considered, we thus expect strongly correlated electron effects with well-defined spin qubits along the $\mathrm{Sc} @ \mathrm{C}_{82}$ chain of the peapods and weak charge fluctuations. Therefore, the system is well characterized by an antiferromagnetic Heisenberg model.

In the semiconducting peapod, we may estimate the hybridization coupling parameters $\gamma_{l k}$ in Eq. (4) from the band splitting at $k=0$, as shown in the circle area in Fig. 4(a). An estimate of $\gamma_{l k}$ can be made by only considering the hybridization interaction between an electron on the fullerene and the same electron in the conduction bands at $k=0$. Assuming $\gamma_{l k=0}=\gamma_{0}$, the same for each conduction band $(l=1,2)$, this approximate Hamiltonian for spin up is

$$
H_{\mathrm{approx}}^{\uparrow}=\epsilon_{0}\left(N_{1 \uparrow}+N_{2 \uparrow}\right)+\varepsilon_{0} n_{\uparrow}+\gamma_{0}\left(c_{1 \uparrow}^{\dagger}+c_{2 \uparrow}^{\dagger}\right) a_{\uparrow}+\text { h.c. },
$$

where $\epsilon_{0}$ is the degenerate energy of the conduction bands and $\varepsilon_{0}$ is the energy of the electron on the fullerene. Let

$$
\begin{aligned}
& \alpha^{\dagger}=\frac{c_{1 \uparrow}^{\dagger}+c_{2 \uparrow}^{\dagger}}{\sqrt{2}}, \\
& \beta^{\dagger}=\frac{c_{1 \uparrow}^{\dagger}-c_{2 \uparrow}^{\dagger}}{\sqrt{2}} .
\end{aligned}
$$

Equation (16) becomes

$$
H_{\mathrm{approx}}^{\uparrow}=\epsilon_{0}\left(\alpha_{\uparrow}^{\dagger} \alpha_{\uparrow}+\beta_{\uparrow}^{\dagger} \beta_{\uparrow}\right)+\varepsilon_{0} n_{\uparrow}+\sqrt{2} \gamma_{0} \alpha_{\uparrow}^{\dagger} \alpha_{\dagger}+\text { h.c. },
$$

and similarly for $H_{\text {approx }}^{\downarrow}$. The eigenenergies are given by $\epsilon_{0}$ and $\operatorname{det}\left(\begin{array}{cc}\epsilon_{0}-E & \sqrt{2} \gamma_{0} \\ \sqrt{2} \gamma_{0} & \varepsilon_{0}-E\end{array}\right)=0$, i.e., $\boldsymbol{\epsilon}_{0}$ and

$$
E_{0}^{ \pm}=\frac{\epsilon_{0}+\varepsilon_{0}}{2} \pm \frac{\epsilon_{0}-\varepsilon_{0}}{2} \sqrt{1+\frac{8 \gamma_{0}^{2}}{\left(\epsilon_{0}-\varepsilon_{0}\right)^{2}}} .
$$

For $\gamma_{0}^{2} \ll\left(\epsilon_{0}-\varepsilon_{0}\right)^{2}$, we obtain the energies at $k=0$ of the bands derived from the LUMO of Sc @ $\mathrm{C}_{82}$ and the first conduction band derived from the nanotube,

$$
E_{0}^{ \pm}=\varepsilon_{0}+\frac{2 \gamma_{0}^{2}}{\epsilon_{0}-\varepsilon_{0}}, \quad \epsilon_{0}-\frac{2 \gamma_{0}^{2}}{\epsilon_{0}-\varepsilon_{0}} .
$$

Hence, the conduction band splitting at $k=0$ is $\delta E=\frac{2 \gamma_{0}^{2}}{\epsilon_{0}-\varepsilon_{0}}$, yielding the coupling energy $\gamma_{0} \simeq 15 \mathrm{meV}$. In the metallic peapod, we may also estimate $\gamma_{l k}$ in Eq. (4) from the anticrossing gaps associated with the nanotube bands and a narrow fullerene band. When $\epsilon_{l k}=E_{k}$, the hybridization energy gap $\Delta E$ is precisely $2 \gamma_{l k}$. For example, the anticrossings shown in the circle regions in Fig. 4(b) yields a coupling energy $\gamma_{l k} \simeq 5 \mathrm{meV}$.

In the metallic peapod, the weak interaction between electrons on the fullerenes and conduction electrons (or holes) in the nanotube, characterized by $\gamma_{l k}$ discussed above, will give rise to Kondo-type coupling between spins on the fullerenes and spins in the metallic nanotube. The energy scale for these couplings is given by $J_{K} \sim \gamma_{l k}^{2} / E_{t}$, where $E_{t}$ is a chargetransfer energy gap, i.e., $E_{t}=E_{0}+U-E_{F}$ or $E_{F}-E_{0} \cdot{ }^{30}$ This gives a typical $J_{K} \sim 0.1 \mathrm{meV}$ for the $(11,11)$ peapod. DFT calculations cannot resolve the difference in $J$ obtained for a $\mathrm{Sc} @ \mathrm{C}_{82}$ chain and the corresponding peapod structure even at a large interfullerene separation. This is consistent with direct exchange dominating RKKY interactions. ${ }^{31}$ Charge fluctuations could be increased by either enhancing the hybridization interaction through a decrease in the fullerenenanotube separation or by tuning the Fermi energy in metallic nanotubes to approach the mixed-valence regime in which the charge-transfer energy tends to zero. This would enhance both the Kondo coupling and the RKKY interaction, in competition with the direct Heisenberg exchange.

\section{CONCLUSION}

In conclusion, $\mathrm{Sc} @ \mathrm{C}_{82}$ peapods offer well-defined spin qubits on the $\mathrm{C}_{82}$ cage, coupled via antiferromagnetic Heisenberg exchange interactions. For the semiconducting case, the upper and lower Hubbard bands of the fullerene 
chain are little affected by the nanotube and occur entirely within the band gap of the nanotube, allowing excitations of the $\mathrm{Sc} @ \mathrm{C}_{82}$ chain independently of the nanotube. Remarkably, for the peapods discussed in this paper, the main function of the nanotubes will be to give mechanical support for the endohedral fullerenes and to protect them from the environment rather than to provide controlled interactions between the spins. An endohedral fullerene peapod thus provides a candidate nanostructure for spin-chain quantum computing. ${ }^{6,7}$

\section{ACKNOWLEDGMENTS}

This work is part of QIP IRC. We thank the Materials Chemistry Consortium, EPSRC-GB (Portfolio Grant No. EP/ D504872) and MML, Oxford for providing the computing facilities. L.G. acknowledges support from the Clarendon Fund and St. Anne's College, Oxford. J.H.J. acknowledges support from the UK MOD and Wolfson College, Oxford. G.A.D.B. thanks EPSRC for financial support (GR/S15808/ 01). We thank S. Benjamin for discussions. *ling.ge@materials.ox.ac.uk

${ }^{1}$ S. C. Benjamin and S. Bose, Phys. Rev. Lett. 90, 247901 (2003).

${ }^{2}$ B. W. Smith, M. Monthioux, and D. E. Luzzi, Nature (London) 396, 323 (1998).

${ }^{3}$ A. Ardavan, M. Austwick, S. C. Benjamin, G. A. D. Briggs, T. J. S. Dennis, A. Ferguson, D. G. Hasko, M. Kanai, A. N. Khlobystov, B. W. Lovett, G. W. Morley, R. A. Oliver, D. G. Pettifor, K. Porfyrakis, J. H. Reina, J. H. Rice, J. D. Smith, R. A. Taylor, D. A. Williams, C. Adelmann, H. Mariette, and R. J. Hamers, Philos. Trans. R. Soc. London, Ser. A 361, 1473 (2003).

${ }^{4}$ R. Kitaura and H. Shinohara, Jpn. J. Appl. Phys., Part 1 46, 881 (2007).

${ }^{5}$ G. W. Morley, B. J. Herbert, S. M. Lee, K. Porfyrakis, T. J. S. Dennis, D. Nguyen-Manh, R. Scipioni, J. van Tol, A. P. Horsfield, A. Ardavan, D. G. Pettifor, J. C. Green, and G. A. D. Briggs, Nanotechnology 16, 2469 (2005).

${ }^{6}$ S. C. Benjamin and S. Bose, Phys. Rev. A 70, 032314 (2004).

${ }^{7}$ J. Fitzsimons, L. Xiao, S. C. Benjamin, and J. A. Jones, Phys. Rev. Lett. 99, 030501 (2007).

${ }^{8}$ J. Baugh, O. Moussa, C. A. Ryan, A. Nayak, and R. Laflamme, Nature (London) 438, 470 (2005).

${ }^{9}$ S. C. Benjamin, A. Ardavan, G. A. D. Briggs, D. A. Britz, D. Gunlycke, J. H. Jefferson, M. A. G. Jones, D. F. Leigh, B. W. Lovett, A. N. Khlobystov, S. A. Lyon, J. J. L. Morton, K. Porfyrakis, M. R. Sambrook, and A. M. Tyryshkin, J. Phys.: Condens. Matter 18, S867 (2006).

${ }^{10}$ Y. Cho, S. Han, G. Kim, H. Lee, and J. Ihm, Phys. Rev. Lett. 90, 106402 (2003).

${ }^{11}$ S. Okada, S. Saito, and A. Oshiyama, Phys. Rev. Lett. 86, 3835 (2001).

${ }^{12}$ T. Okazaki, T. Shimada, K. Suenaga, Y. Ohno, T. Mizutani, J. Lee, Y. Kuk, and H. Shinohara, Appl. Phys. A: Mater. Sci. Process. 76, 475 (2003).
${ }^{13}$ M. Otani, S. Okada, and A. Oshiyama, Phys. Rev. B 68, 125424 (2003).

${ }^{14}$ O. Dubay and G. Kresse, Phys. Rev. B 70, 165424 (2004).

${ }^{15}$ M. H. Du and H. P. Cheng, Phys. Rev. B 68, 113402 (2003).

${ }^{16}$ J. Lu, S. Nagase, S. Zhang, and L. Peng, Phys. Rev. B 68, 121402(R) (2003).

${ }^{17}$ J. Lu et al., Phys. Rev. B 71, 235417 (2005).

${ }^{18}$ R. Saito, G. Dresselhaus, and M. S. Dresselhaus, Physical Properties of Carbon Nanotubes (Imperial College Press, London, 1998).

${ }^{19}$ A. D. Becke, Phys. Rev. A 38, 3098 (1988).

${ }^{20}$ A. D. Becke, J. Chem. Phys. 98, 5648 (1993).

${ }^{21}$ C. Lee, W. Yang, and R. G. Parr, Phys. Rev. B 37, 785 (1988).

${ }^{22}$ R. Dovesi, V. R. Saunders, C. Roetti, R. Orlando, C. M. Zicovich-Wilson, F. Pascale, B. Civalleri, K. Doll, N. M. Harrison, I. J. Bush, Ph. D’Arco, and M. Llunell, CRYSTALO6 Users Manual (University of Torino, Torino, 2006).

${ }^{23}$ H. B. Schelegel, J. Comput. Chem. 3, 214 (1982).

${ }^{24}$ R. S. Mulliken, J. Chem. Phys. 23, 1833 (1955).

${ }^{25}$ J. Lu, X. Zhang, X. Zhao, S. Nagase, and K. Kobayashi, Chem. Phys. Lett. 332, 219 (2000).

${ }^{26}$ W. Harneit, Phys. Rev. A 65, 032322 (2002).

${ }^{27}$ J. A. Chan, B. Montanari, J. D. Gale, S. M. Bennington, J. W. Taylor, and N. M. Harrison, Phys. Rev. B 70, 041403(R) (2004).

${ }^{28}$ Y. Ito, W. Fujita, T. Okazaki, T. Sugai, K. Awaga, E. Nishibori, M. Takata, M. Sakata, and H. Shinohara, ChemPhysChem 8, 1019 (2007).

${ }^{29}$ D. G. Pettifor, Bonding and Structure of Molecules and Solids (Oxford University Press, Oxford, UK, 1995).

${ }^{30}$ A. C. Hewson, The Kondo Problem to Heavy Fermions (Cambridge University Press, Cambridge, 1993).

${ }^{31}$ M. A. Ruderman and C. Kittel, Phys. Rev. 96, 99 (1954). 